\title{
La acción social profesional del Trabajo Social en el área de justicia juvenil en Chile
}

\author{
Karina Aedo Poblete ${ }^{1}$
}

\section{RESUMEN}

El año 2007 se implementa en Chile la Ley de Responsabilidad Penal Adolescente, llevando a cabo distintos programas centrados en el control social, los cuales han sido ejercidos históricamente por trabajadores sociales. Esto ha llevado a repensar la acción profesional, en la que se evidencian distintas tensiones; por una parte, un sistema enfocado en que los adolescentes infractores de ley lleven a cabo sus medidas y sanciones respecto de los delitos cometidos y que frente a esto logren la reinserción social, pero, por otra, la invisibilización de un sistema neoliberal, altamente desigual y violento, en el que se responsabiliza únicamente a los jóvenes y a sus familias. Es decir, un sistema que vulnera y pasa por alto todo tipo de derecho, que a través de políticas públicas de reinserción social nos hace creer que encuentra la solución respecto de las mismas problemáticas que este ha creado desde el surgimiento de la cuestión social.

Palabras claves: políticas de reinserción, trabajo social crítico, cuestión social, justicia juvenil

\section{Professional social action through social work in the child justice system in Chile}

In 2007 Chile implemented its Law on Adolescent Criminal Liability, bringing to fruition a number of programs centering on social control, which have historically been undertaken by social workers. This has led to a rethinking of professional actions, showing a range of different tensions: firstly, a system focused on ensuring that adolescent offenders complete the measures and sanctions imposed for the crimes they have committed, and that they then achieve reinsertion into society, contrasted with a tendency to conceal a severely unequal and violent neoliberal system that attributes all responsibility to young people and their families. Thus, a system that breaches

Chilena, Trabajadora Social, trabajando actualmente en el Programa de Salidas Alternativas Wekintün, de la Corporación CIEM de Villarrica. E-mail: kaedo. poblete@gmail.com 
La acción social profesional del Trabajo Social en el área de justicia juvenil en Chile / Aedo

and overlooks all types of rights, that adopts public policies for reinsertion into society in a bid to claim that a solution has been found for the same problems that it has itself created through the formation of the social issue.

Keywords: reinsertion policies, critical social work, social issue, juvenile justice.

\section{A ação social profissional do Serviço Social na área da justiça juvenil no Chile}

\section{RESUMO}

No ano 2007 é implementada no Chile a Lei de Responsabilidade Penal Adolescente, com a realização de distintos programas centrados no controle social, os quais têm sido exercidos historicamente por assistentes sociais. Isto conduziu a um repensar da ação profissional, no qual se evidenciam distintas tensões. Por um lado, um sistema focado em que os adolescentes infratores cumpram suas medidas e sanções a respeito dos delitos cometidos e que frente a isto consigam a reinserção social, mas, por outro lado, a invisibilização de um sistema neoliberal, altamente desigual e violento, no qual se responsabiliza unicamente os jovens e suas famílias. Isto quer dizer, um sistema que vulnera e ignora todo tipo de direito, que através de políticas públicas de reinserção social nos faz acreditar que encontra a solução para as mesmas problemáticas que este tem criado desde o surgimento da questão social.

Palavras-chave: políticas de reinserção, trabalho social crítico, questão social, justiça juvenil.

\section{Introducción}

Con la implementación de la Ley 20.084, o más conocida como la Ley de Responsabilidad Penal Adolescente (LRPA), surgen distintos programas enfocados en el control social y en la reinserción social ${ }^{2}$.

Cabe destacar que, anterior a dicha ley, existía cierta irregularidad en cuanto a las medidas o condenas que recibirían los adolescentes que habían cometido algún delito, debiendo ser sometidos a

2 Sobre la base de la última cuenta pública realizada por el Servicio Nacional de Menores (2018), durante 2017 fueron atendidos 14.177 NNA y sus familias en el área de responsabilidad penal, representando un $28 \%$ del total de atendidos por SENAME. 
una declaración de discernimiento y, luego, era el juez quien dictaba si los adolescentes serían tratados como adultos ante la ley o bien serian ingresados junto con su familia a una medida de protección (SENAME, s/f).

Esto llevaba a que existiera cierta confusión en cuanto a las consecuencias por cometer actos delictivos en la adolescencia, donde las diferencias eran desde ser tratados como adultos (sistema penal), hasta no reconocer dichos delitos, centrando el problema en las familias (sistema de protección).

Por lo tanto, la Ley 20.084 dio paso a un nuevo campo de acción, enfocado en que los profesionales generen espacios de reflexión individual que permitan el cambio en el adolescente, lo que implica un escaso trabajo con las familias y una fuerte gestión de redes para derivar al joven según los distintos "problemas" identificados (consumo, deserción escolar, salud, entre otros).

A su vez, trabajar en el área de justicia juvenil ha implicado repensar la forma de entender el problema y, finalmente, cómo se llevará a cabo la intervención.

Con base en lo mismo podemos apreciar que, al trabajar el tema de la delincuencia, "(...) no consideran la complejidad e interrelación de los problemas que circundan la vida cotidiana, sino más bien hacen una sumatoria de condiciones que etiquetan a estos niños y niñas como complejos, situando el problema en ellos y ellas mismos" (Muñoz \& Aguirre, 2012, p. 54).

De la reciente cita extraemos dos elementos centrales: primero, la complejidad e interrelación que se da entre distintos temas, pero que podríamos denominar como las distintas manifestaciones de la cuestión social (Rozas, 2009b) y, por otra parte, las denominadas "etiquetas".

Frente a esto, es importante resaltar que, por mucho tiempo, el "problema" de la delincuencia se ha observado a nivel microsocial (Montaño, 2007), primeramente centrado en el adolescente y en segundo lugar en su familia. 
La acción social profesional del Trabajo Social en el área de justicia juvenil en Chile / Aedo

Es necesario que los profesionales que intervienen en el área de justicia juvenil logren comprender las distintas contradicciones que se producen en este espacio, las cuales son respuesta a un modelo altamente desigual, basado en la producción en una economía capitalista y neoliberal.

Al comprender estos elementos podemos comenzar a buscar las formas de dar a conocer estas tensiones y dejar de "etiquetar" al adolescente como un delincuente que, de manera fortuita, ha llegado a cometer ciertos delitos.

Por lo tanto, se trata de observar al adolescente en su contexto, donde ha sido invisibilizado desde su temprana infancia a través de la vulneración de sus derechos básicos, como el derecho a una educación y atención de salud de calidad, vivienda, entre otros.

Para dar respuesta a estas situaciones que desde el Estado han sido denominadas como "problemas sociales" (Rozas, 2009a) se han creado distintas políticas que, desde el área de justicia juvenil, han sido denominadas como "políticas de reinserción social".

Según el Ministerio de Justicia, se entiende la reinserción social como un "proceso sistemático de acciones que se inician desde que se dicta una sentencia, durante el periodo del cumplimiento de la condena y prosigue cuando la persona retorna a su vida en sociedad" (Ministerio de Justicia, 2015). Una vida en sociedad que ha ocultado la cuestión social y sus distintas manifestaciones, y que exige un estilo de vida estandarizado para todos los adolescentes.

Es decir, el mismo sistema que los ha excluido de la sociedad no permite el desarrollo integral de estos en su vida cotidiana, debido a que establece una única forma para la diversidad de jóvenes presentes, en la que se aprecia como fundamental la intervención profesional.

Según Rozas (2009a), en las políticas públicas, la intervención profesional se constituye como uno de los ejes centrales en cuanto a la ejecución de las mismas. Por esto la importancia de reflexionar respecto de la labor profesional en la intervención. 
Sobre la base de lo expuesto, en este artículo se discutirá sobre las contradicciones en cuanto a la intervención profesional del trabajador social, haciendo distinciones en cuanto al asistente social operador de las políticas públicas y las orientaciones técnicas, y un trabajador social crítico, consciente de dichas contradicciones y que enfoca su intervención de manera contraria a lo impuesto.

Debido a lo mismo se cuestionará la relación entre reinserción social y mecanismos de dominación instaurados por el sistema imperante.

\section{La reinserción social como mecanismo de dominación}

La ley de Responsabilidad Penal Adolescente (LRPA) surge en Chile como respuesta a los problemas para distinguir las penas o sanciones que se aplicarían frente a la comisión de un delito efectuado por jóvenes y adolescentes desde los 14 a los 18 años de edad.

Según lo explicado por el Servicio Nacional de Menores, “...esta legalidad creó un sistema de justicia juvenil especializado, pensando en la reinserción social de adolescentes y jóvenes..." SENAME (s.f.). Es aquí cuando la temática centrada a nivel microsocial (Montaño, 2007) en adolescentes/jóvenes infractores de ley, nos lleva a repensar cuáles son las dificultades o problemáticas que existen en la base y que, por lo tanto, según este sistema, exigiría la reinserción social como solución.

Se debe considerar que la reinserción social, según Womith (citado por Ahumada \& Grandón, 2015), se enfoca principalmente en las estrategias e intervenciones que facilitan el cambio en el adolescente, esperando que estos puedan tener una vida satisfactoria.

Según lo anterior, se puede plantear la siguiente pregunta: ¿el sistema neoliberal actual facilita que las personas puedan tener un nivel de vida satisfactorio? 
La acción social profesional del Trabajo Social en el área de justicia juvenil en Chile / Aedo

Frente a esto, nos encontramos con una realidad que ha sido ocultada por parte del sector dominante, quienes han insistido en que la responsabilidad recae en el sujeto (infractor de ley), pero no en las distintas situaciones de desigualdad e injusticia social histórica que éste ha vivido, como consecuencia del modelo de producción imperante (Vivero, 2016).

Se requiere contextualizar acerca de los elementos históricos que han evidenciado las distintas situaciones de desigualdad social, las cuales han involucrado directamente a jóvenes y sus familias, considerando que la mayoría de estos son de sectores sociales en situación de pobreza ${ }^{3}$, lo cual, según Zambrano \& Pérez (2004), se consideraría como un obstáculo para las personas, porque han sido vulneradas en sus derechos más básicos.

Por lo cual se requiere pasar desde una mirada centrada en lo punitivo a una restaurativa de derechos, que permita que los adolescentes y sus familias puedan obtener respuestas frente a sus necesidades básicas.

De acuerdo con lo anterior, Montaño (2007) plantea que las políticas públicas dan respuesta a "necesidades individuales, diferentes según el poder adquisitivo de cada quien. Por tanto, tales respuestas no constituirían derecho sino (...) un servicio comercializable" (p. 5). Es necesario comprender que un alto porcentaje de los jóvenes ingresados a programas de la LRPA han pasado por el área de protección, es decir, han sido vulnerados en sus derechos. En muchos casos, esto dificulta el acceso a mejorar y construir una realidad distinta a la vivida, y cambiarla implicaría un mayor poder adquisitivo que, en su contexto y a un nivel macrosocial, el sistema no permite que estos lo generen.

3 Según la última evaluación de desempeño realizada por el Programa de Salidas Alternativas Wekintün de la corporación CIEM Villarrica (2019), se puede apreciar que justamente una de las dificultades para la intervención se relaciona con el "contexto familiar de riesgo", lo que en dicho documento se explica como las situaciones de vulnerabilidad vividas por los adolescentes y las dificultades de acceso a educación, salud y programas de tratamiento en caso de ser necesarios, limitando la intervención en dichas áreas. 
Aquí es cuando nos encontramos con un Estado que aparentemente quiere dar respuesta a estas situaciones, basando su ayuda en lo que ellos visualizan como los distintos "problemas sociales" (Rozas, 2009a). No es extraño encontrarnos con la creación de otros programas, que finalmente buscan solucionar una parte de lo que ellos mismos han generado y definido como "problema".

Siguiendo a la misma autora, quien realiza la distinción entre la cuestión social y la interpretación (consciente) que ha hecho el Estado capitalista, en no reconocerla y finalmente considerarla de manera fragmentada en "problemas sociales", es que se debe tener presente esta asignación de significado para comprender las contradicciones que dicho sistema no resuelve (Rozas, 2009b).

Finalmente, y reafirmando lo planteado, podemos ver que el Estado inmerso en un sistema neoliberal espera que las políticas públicas puedan solucionar "problemas" que define como transitorios o estáticos, los cuales, en el ámbito de la justicia juvenil, implican mucho más que aspectos que se pueden solucionar de manera rápida (Rozas, 2009b) ${ }^{4}$.

Para esto debemos detenernos y pensar cuáles han sido los elementos que han estado históricamente involucrando a los jóvenes y sus familias en situaciones de desigualdad e injusticia social (Vivero, 2016).

\section{La acción profesional del Trabajo Social en el área de justicia juvenil}

Luego de haber expuesto las contradicciones presentes en la LRPA y las políticas de reinserción social, es importante reflexionar acerca de la acción profesional que se realiza en este campo.

Nos encontramos aquí con una gran contradicción en cuanto a los tiempos definidos por la política pública (el cual suele ser limitado y establecido a priori) y lo que realmente las familias necesitan, generando tensiones profesionales, en las cuales la realidad y las personas con las que se trabaja de manera directa no se encuentran sujetas solamente al tiempo actual, sino a lo pasado, presente y futuro. 
La acción social profesional del Trabajo Social en el área de justicia juvenil en Chile / Aedo

Frente a estos dilemas, la profesión no se ha visto exenta, ya que, finalmente, quienes aplican la política pública basados en sus orientaciones técnicas han sido trabajadores sociales, los cuales, desde una lógica más tradicional y respondiendo acorde a lo que el sistema impone, realizan intervenciones desde un rol de control social (Vivero, 2010).

Según Rozas (2009a) “...existe un consenso colectivo profesional respecto a la relación entre cuestión social, políticas sociales e intervención profesional" (p. 1). Por lo tanto, existe un modelo económico de dominación, que no solo define las acciones de los sectores excluidos, sino también la acción e intervención profesional.

Por otra parte, Montaño (2007) explica que existe una contradicción respecto de la intervención que se lleva a cabo, lo cual analiza de la siguiente manera:

El Servicio Social desarrolla su intervención en un espacio de tensión y contradicción, entre su funcionalidad con la reproducción del sistema (a partir de los intereses hegemónicos del gran capital) y la defensa de derechos y conquistas sociales (a partir de demandas y luchas de las clases trabajadoras y subalternas). (p. 3)

Según los planteamientos teóricos utilizados para este artículo, podríamos definir dos tipos de profesionales, por una parte, el/ la asistente social tradicional y, por otra, un/una trabajador/a social con perspectiva crítica de la realidad (Montaño, 2007; Rozas, 2009a, 2009b; Vivero, 2016) . $^{5}$

Respecto del asistente social tradicional, podemos mencionar que, en el área de justicia juvenil, se desempeñaría desde el control social y desde la resolución de "problemas sociales" indivi-

Ambos tipos de profesionales descritos permiten apreciar las diferencias teóricas y prácticas al momento de abordar la intervención. Si bien éstas no son rígidas, de todas formas permiten distinguir entre una práctica centrada solamente en lo que política estipula y en otra que, si bien cumple ciertos estándares, no deja de lado aspectos esenciales para comprender el contexto político y sociohistórico de los sujetos. 
duales. Su análisis consiste en buscar en el adolescente la causa de sus conductas contranormativas y, cuando no logra hallarla, recurre a buscar el "problema" en la familia, que de una forma u otra influenció en los comportamientos de este.

Por lo tanto, este tipo de profesional no es consciente de la implementación de un modelo neoliberal dominante que ha establecido las reglas del juego (Rozas, 2009a), otorgando la responsabilidad a los propios sujetos.

Además, en la práctica se puede apreciar que en las intervenciones “...no se asume la desigualdad y las injusticias sociales como consecuencia estructural del modelo de producción" (Vivero, 2016, p. 127), sino que se mantiene la aplicación de instrumentos estandarizados que detectan los riesgos a los cuales se expone el joven desde su contexto microsocial y no desde la estructura en la cual se encuentra inmerso.

Frente a esto surge la necesidad de volver a mirar la acción profesional y transformar esas prácticas instrumentales, que además funcionan con una lógica de dominación, en un hacer profesional que comprenda las distintas manifestaciones de la cuestión social - que han provocado finalmente los actos delictuales-, pero ahora desde una historia de desigualdad, que se sigue manteniendo en el nivel de la intervención.

Ahora bien, se debe destacar la intervención que realizan los/las trabajadores sociales con perspectiva crítica de la realidad, los cuales, según Vivero (2016), deben asumir la realidad histórica y política en la cual se encuentran los actores sociales.

Rozas (2009a) agrega que la perspectiva crítica "reposiciona al profesional, en cuanto éste debe incorporarse en una lectura teórica y crítica que le posibilita resignificar la demanda en el plano analítico..." (p. 12). Por lo tanto, el/la trabajador/a social debe mantener una postura teórica crítica, que fundamente su acción y que permita visualizar las desigualdades presentes en la realidad, y que desde la historia se ha querido invisibilizar. 
Finalmente, es necesario agregar lo que Montaño (2007) nos explica sobre la labor de politización del profesional: "Politizar la práctica profesional significa, primeramente, desvelar, explicitar, descubrir, las contradicciones de intereses, las tensiones (manifiestas o latentes, explícitas o implícitas), existentes entre los sujetos/actores de las realidades donde interviene el profesional" (p. 10).

Frente a lo expuesto, el profesional debe estar atento a desvelar dichas contradicciones y permitir que los actores sociales comprendan estas situaciones, con el fin de generar procesos de reflexión que posibiliten la superación del sistema capitalista neoliberal actual.

\section{Conclusiones}

Para la intervención en el área de justicia juvenil, podemos concluir y aportar lo siguiente:

Primero, la contradicción que se produce en el trabajo con los adolescentes que han cometido infracciones, las cuales, desde el Estado, han sido "solucionadas" o más bien disminuidas mediante la división de distintos "problemas sociales".

En la práctica estas intervenciones se realizan como ayuda en temas de salud, frente a situaciones de deserción escolar, consumo problemático de drogas, situación de pobreza, entre otras, las cuales finalmente responden a las distintas manifestaciones de la cuestión social (Rozas, 2009b).

Además, en la práctica esto se traduce en la intervención de distintos programas que trabajan con el adolescente y su familia, en los que un mismo joven puede estar en tres o cuatro programas de forma paralela, lo que puede provocar la sobreintervención respecto de los problemas definidos desde las políticas públicas.

Segundo, la responsabilización que se otorga a nivel individual o, dicho en palabras de Montaño (2007), las distinciones entre lo micro y macrosocial. Además, esto ha llevado a buscar "culpables" 
frente a las conductas de los jóvenes, los cuales suelen centrarse en la familia y en las funciones que estas han ejercido desde la primera infancia del joven.

Se ve a la familia desde un modelo ideal, ya que, según Rodríguez (2013), “...se habla de la familia en un sentido homogéneo, universal, se hace referencia a este tipo específico de familia sin reconocer la heterogeneidad nacida desde la diversidad cultural..." (p. 127). Y esto porque, dentro de la lógica de los programas de la LRPA, los adolescentes deberían reinsertarse a la sociedad de una forma homogénea y no desde su diversidad, proveniente esta de la diferencias que existen en sus espacios familiares y estilos de crianza.

Por otra parte, según Vivero (2016), “....no se reconoce en lo absoluto que los sujetos, si bien construyen la historia, no lo hacen en la nada, sino a partir de las condiciones materiales y simbólicas en las cuales desarrollan la vida cotidiana" (p. 137). Esto a raíz de que, finalmente, los sujetos y las familias no construyen solamente respecto de sus propios intereses, sino que existen condiciones en las cuales se han desarrollado que muchas veces se mantienen en relación al sistema en el cual están inmersos.

Tercero y último, repensar la acción profesional del Trabajo Social en esta área, ya que se necesita, más que aplicación de instrumentos, que definen y categorizan las características de los adolescentes, de una transformación.

Respecto de lo que implicaría ser un/una trabajador/a social crítico, Rozas (2009a) nos plantea:

En tal sentido, nuestra perspectiva teórica sobre la intervención reposiciona al profesional, en tanto éste debe incorporar una lectura teórica y crítica que le posibilita resignificar la demanda en el plano analítico, en sus dimensiones no sólo materiales, sino, sobre todo, en la comprensión de la degradación humana a la que se ha sometido la vida de los sujetos. (p. 12)

Finalmente, mantener una postura profesional a partir de la corriente crítica desafía al trabajo en equipo, principalmente por 
La acción social profesional del Trabajo Social en el área de justicia juvenil en Chile / Aedo

aquellos que continúan replicando las acciones delimitadas en las políticas públicas y que se resisten al cambio.

Frente a esto, Vivero (2010) nos dice: “... la conciencia social crítica es vista como algo negativo, obstaculizador del desarrollo y por lo tanto lo que se privilegia es el consenso" (p. 427). Por lo tanto, se puede agregar que para este sistema el consenso es cómodo, es ciego, ya que no permite ver la realidad, pero justamente el desafío profesional es dar a conocer las contradicciones presentes en nuestros campos de acción, aquello que afecta a los actores sociales, pero también delimitan la intervención y no permiten dar a conocer situaciones estructurales que afectan y reproducen modelos de dominación.

\section{Bibliografía}

Ahumada, H. y Grandón, P. (2015). Significados de la reinserción social en funcionarios de un centro de cumplimiento penitenciario. Psicoperspectivas, 14(2), 84-95.

Ministerio de Justicia. (2015). ¿Cómo entendemos la reinserción social? Recuperado el 15 de diciembre de 2016 de http://www. minjusticia.gob.cl/reinsercion-social/que-es-la- reinsercion/

Montaño, C. (2007). Trabajo social e intervención: la politización de la acción profesional. Recuperado el 30 de agosto del 2018 de http://www.ts.ucr.ac.cr/binarios/pela/pl- 000360.pdf

Muñoz, C. \& Aguirre, B. (2012). Política Nacional de Infancia en Chile: Derechos Limitados. Portularia, XII(1), 49-58.

Rodríguez, X. (2013). Estado, familias y saberes para resignificar políticas públicas. Rumbos TS, VII(7), 123-132.

Rozas, M. (2009a). ¿Cómo asumir el estudio de la cuestión social y las políticas sociales en la formación profesional en Trabajo Social? Recuperado el 25 de agosto del 2018 de http://www.ts.ucr. ac.cr/binarios/congresos/reg/slets/slets-018-007.pdf

Rozas, M. (2009b). Tendencias teórico-epistemológicas y metodológicas en la formación profesional. Recuperado el 10 de septiembre del 2018 de http://www.ts.ucr.ac.cr/binarios/congresos/reg/slets/slets-018- 006.pdf

SENAME. (2018). Cuenta Pública Participativa 2018. Recuperado el 05 de agosto de 2019 de http://www.sename.cl/web/cuentapublica-participativa-2018/ 
SENAME. (2019). Evaluación anual de desempeño, Programa de Salidas Alternativas Wekintün. Villarrica: Centro de Iniciativa Empresarial CIEM Villarrica.

SENAME. (s/f). MARCO LEGAL Ley de Responsabilidad Penal Adolescente. Recuperado el 03 de septiembre de 2018 de http:// www.sename.cl/web/marco-legal-ley- responsabilidadpenal-adolescente/

Vivero, L. (2010). Las políticas públicas como práctica de contrainsurgencia social. Revista de ciencias sociales, XVI (3), 418-429.

Vivero, L. (2016). Influencia del neoliberalismo en el trabajo social chileno: discursos de profesionales y usuarios. Revista colombiana de Ciencias Sociales, 8(1), 125-148.

Zambrano, A. \& Pérez, R. (2004). Construcción de identidad en jóvenes infractores de ley, una mirada desde la psicología cultural. Revista de psicología de la Universidad de Chile, XIII(1), 115-132. 